\title{
Indicadores antropométricos e riscos à saúde da pessoa com deficiência em uma cidade do sul de Minas
}

\author{
Luís Paulo Pires Corrêa, Deyliane Aparecida de Almeida Pereira
}

\begin{abstract}
Resumo
O sedentarismo e o hábito alimentar irregular ocasiona o aumento de peso e de gordura corporal no indivíduo, tornando-o mais suscetível a diversas enfermidades, portanto, os indicadores antropométricos contribuem para a predição de possíveis agravos e riscos a saúde das pessoas. Este estudo tem por objetivo identificar e analisar as prevalências e associações dos riscos à saúde das pessoas com deficiência por meio dos indicadores antropométricos (IA). Trata-se de um estudo quantitativo de corte transversal, com característica descritiva, sendo a amostra composta por 23 (correspondente a $69,7 \%$ da população deficiente do município) pessoas com deficiência de ambos os sexos, residentes em uma cidade localizada no sul de Minas Gerais. Para coleta de dados foi aferido as medidas antropométricas: massa corporal, estatura, perímetro da cintura e dobras cutâneas, para o cálculo dos indicadores. Pela análise dos dados verifica-se que a idade média é de 41 anos (DP \pm 14$)$ e Percentual de Gordura corporal médio de 35,63 (DP $\pm 9,30)$, sendo que 65,2\% encontram-se com excesso de gordura corporal de acordo com a classificação do Índice de Massa corporal (IMC), já pelo Índice de Adiposidade Corporal (IAC) 85,7\% apresentam este quadro. Quando analisado a Razão Cintura Quadril (RCQ) e Relação Cintura/estatura (RCE) 91,3\% das pessoas com deficiência apresentam risco para o desenvolvimento de doenças crônicocardiovasculares. Os resultados apresentados neste estudo demonstram altas prevalências de obesidade, sobrepeso e de risco para possíveis doenças crônico-degenerativas devido à falta de hábitos saudáveis de vida, como a prática de atividade física e o controle alimentar adequado, o que pode ocasionar em grande incidência a agravos e gastos em saúde. Portanto, ressalta-se a importância da criação de estratégias em políticas públicas no município para o incentivo da prática de atividades físicas para pessoas com deficiência e de reeducação alimentar com profissionais capacitados e, observa-se a relevância do uso de indicadores antropométricos por ser um método simples, de baixo custo e facilmente aplicável a um grande grupo de pessoas para diagnosticar problemas em saúde pública como aumento da gordura corporal e fatores associados.
\end{abstract}

Descritores: Pessoa com Deficiência; Indicadores Antropométrico; Riscos à saúde. 